\section{BMJ Open \\ Respiratory \\ Research}

\title{
Pulmonary artery perfusion versus no pulmonary perfusion during cardiopulmonary bypass in patients with COPD: a randomised clinical trial
}

\author{
Katrine B Buggeskov, ${ }^{1}$ Martin M Sundskard, ${ }^{1}$ Thomas Jonassen, ${ }^{2}$ \\ Lars W Andersen, ${ }^{1}$ Niels H Secher, ${ }^{3}$ Hanne B Ravn, ${ }^{1}$ Daniel A Steinbrüchel, ${ }^{4}$ \\ Janus C Jakobsen, ${ }^{5,6}$ Jørn Wetterslev ${ }^{5}$
}

To cite: Buggeskov KB, Sundskard MM, Jonassen T, et al. Pulmonary artery perfusion versus no pulmonary perfusion during cardiopulmonary bypass in patients with COPD: a randomised clinical trial. BMJ Open Resp Res 2016:3:e000146.

doi:10.1136/bmjresp-2016000146

- Additional material is available. To view please visit the journal (http://dx.doi.org/ 10.1136/bmjresp-2016000146)

Received 9 June 2016 Revised 14 July 2016 Accepted 15 July 2016

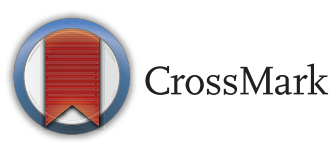

For numbered affiliations see end of article.

Correspondence to Dr Katrine B Buggeskov; katrine.buggeskov@gmail. com

\section{ABSTRACT}

Introduction: Absence of pulmonary perfusion during cardiopulmonary bypass (CPB) may be associated with reduced postoperative oxygenation. Effects of active pulmonary artery perfusion were explored in patients with chronic obstructive pulmonary disease (COPD) undergoing cardiac surgery.

Methods: 90 patients were randomised to receive pulmonary artery perfusion during CPB with either oxygenated blood $(n=30)$ or histidine-tryptophanketoglutarate (HTK) solution $(n=29)$ compared with no pulmonary perfusion $(n=31)$. The coprimary outcomes were the inverse oxygenation index compared at 21 hours after starting CPB and longitudinally in a mixed-effects model (MEM). Secondary outcomes were tracheal intubation time, serious adverse events, mortality, days alive outside the intensive care unit (ICU) and outside the hospital.

Results: 21 hours after starting CPB patients receiving pulmonary artery perfusion with normothermic oxygenated blood had a higher oxygenation index compared with no pulmonary perfusion (mean difference (MD) $0.94 ; 95 \% \mathrm{Cl} 0.05$ to $1.83 ; p=0.04$ ). The blood group had also a higher oxygenation index both longitudinally (MEM, $p=0.009$ ) and at 21 hours (MD 0.99; Cl 0.29 to 1.69; $\mathrm{p}=0.007$ ) compared with the HTK group. The latest result corresponds to a difference in the arterial partial pressure of oxygen of $23 \mathrm{~mm} \mathrm{Hg}$ with a median fraction of inspired oxygen of 0.32. Yet the blood or HTK groups did not demonstrate a longitudinally higher oxygenation index compared with no pulmonary perfusion (MEM, $\mathrm{p}=0.57$ and 0.17 ). Similarly, at 21 hours there was no difference in the oxygenation index between the HTK group and those no pulmonary perfusion (MD $0.06 ; 95 \% \mathrm{Cl}-0.73$ to $0.86 ; p=0.87$ ). There were no statistical significant differences between the groups for the secondary outcomes.

Discussion: Pulmonary artery perfusion with normothermic oxygenated blood during cardiopulmonary bypass appears to improve postoperative oxygenation in patients with COPD undergoing cardiac surgery. Pulmonary artery

\section{KEY MESSAGES}

Although the technical refinement of cardiopulmonary bypass (CPB) has progressively improved the surgical outcomes of heart diseases, postoperative CPB-induced lung dysfunction still remains an important contributing factor to increased morbidity and mortality, especially for patients with chronic obstructive pulmonary disease (COPD).

For patients with COPD undergoing cardiac surgery this trial shows that pulmonary artery perfusion with normothermic oxygenated blood during CPB improves postoperative oxygenation. This trial outlines strengthened evidence for pulmonary artery perfusion with blood during CPB with the need for a larger confirmatory trial to change clinical practice of CPB for patients with COPD undergoing cardiac surgery.

perfusion with hypothermic HTK solution does not seem to improve postoperative oxygenation.

Trial registration number: NCT01614951; Pre-results.

\section{INTRODUCTION}

Pulmonary dysfunction is one of the leading causes of prolonged hospitalisation, morbidity and mortality after cardiopulmonary bypass (CPB)-dependent cardiac surgery and varies from only a short need of mechanical ventilation to fever, productive cough, pulmonary oedema, respiratory failure and in the most severe cases adult respiratory distress syndrome. ${ }^{1-5}$ The aetiology is multifactorial and can be separated into poor postoperative lung mechanics and abnormal gas exchange. However, CPB is considered to be the main contributor to postoperative 
pulmonary dysfunction due to (1) contact of blood with the heart-lung machine's artificial surfaces, (2) perfusion of only the systemic circulation and not the pulmonary circuit with non-physiological laminar flow, resulting in (3) release of endotoxins from the splanchnic area, and after weaning from CPB (4) warm ischaemic reperfusion injury of especially the lungs, all leading to activation of the inflammatory response and consequently interstitial pulmonary oedema. ${ }^{6-8}$

The lungs have a dual blood supply from the pulmonary and bronchial arteries with extensive collateral connections. Blood supply to the lungs during CPB is limited to flow through the bronchial arteries and an ischaemic injury occurs as bronchial blood flow is insufficient to meet the lungs metabolic demands. It has now been confirmed in several animal models that bronchial artery blood flow is decreased substantially during CPB, and although exposure to CPB alone is enough to cause pulmonary injury, concomitant cessation of pulmonary artery flow contributes significantly to the development of postoperative pulmonary dysfunction. ${ }^{9-12}$

The results of animals studies, ${ }^{13-16}$ small randomised trials in infants with congenital heart disease $\mathrm{e}^{17-20}$ and inconclusive minor randomised trials and observational studies in adults ${ }^{21-25}$ indicates that pulmonary artery perfusion with normothermic oxygenated blood preserves postoperative oxygenation. Further, lung transplantation experiences has shown that pulmonary artery perfusion with a hypothermic preservation solution protects the lungs from warm ischaemic injury during storage and transplantation. ${ }^{26}$ We therefore hypothesised that pulmonary artery perfusion with normothermic oxygenated blood or uniform hypothermia by pulmonary artery perfusion with a hypothermic preservation solution potentially can attenuate the warm ischaemic injury to the lungs during CPB.

The aim of our trial was therefore to investigate two different concepts of pulmonary artery perfusion during $\mathrm{CPB}$ in a patient population with an increased risk of lung injury due to chronic obstructive pulmonary disease (COPD). ${ }^{27}$ We wanted to test two hypotheses: (1) pulmonary artery perfusion with normothermic oxygenated blood and (2) cooling of the lungs by pulmonary artery perfusion with hypothermic histidine-tryptophanketoglutarate (HTK) solution improves postoperative oxygenation in patients with COPD undergoing cardiac surgery compared with (3) no pulmonary artery perfusion.

\section{METHODS}

The Pulmonary Protection Trial was approved by the Committees on Biomedical Research Ethics of The Capital Region of Denmark, the Danish Medicines Agency and the Danish Data Protection Agency and registered at ClinicalTrials.gov (6 June 2012, NCT01614951, Principal Investigator Daniel A Steinbrüchel). Written informed consent was obtained from each patient prior to randomisation and the trial was conducted in accord with the ethical standards of the Helsinki Declarations of $1975 .^{28}$

The rationale for and design of the trial including the detailed statistical analysis plan have been published. ${ }^{27} 29$ The methodology is summarised in the following paragraph and adheres to Equator guidelines.

\section{Trial design}

The Pulmonary Protection Trial was a randomised, parallel group, participants, statistician and conclusion drawers blinded trial. The good clinical practice unit at Copenhagen University Hospital monitored adherence to the protocol and data registration. The steering group vouches for the accuracy, completeness and statistical analysis of the data, and for the adherence of this report to the trial protocol and statistical analysis plan. ${ }^{27} 29$

\section{Patients}

We screened patients 18 years of age or older with COPD admitted to the Department of Cardiothoracic Surgery, Rigshospitalet, Denmark for coronary artery bypass grafting, aortic valve replacement or the two procedures combined. COPD was diagnosed in patients with an irreversible airway obstruction (forced expiratory volume in $1 \mathrm{~s}\left(\mathrm{FEV}_{1} /\right.$ forced vital capacity $\left.<0.7\right)$ and classified (mild, moderate, severe and very severe) in accordance with GOLD. ${ }^{30}$ The main exclusion criteria were previous heart or lung surgery, previous thoracic radiation, left ventricular ejection fraction $<20 \%$, tracheal intubation or medically treated pneumonia immediately prior to surgery.

\section{Randomisation}

Patients were assigned in a 1:1:1 ratio to one of three interventions (1) pulmonary artery perfusion with normothermic oxygenated blood, (2) pulmonary artery perfusion with hypothermic HTK solution or (3) no pulmonary artery perfusion during CPB. Randomisation was performed centrally, on a website hosted by The Copenhagen Trial Unit, by a computer-generated allocation sequence in permuted blocks of varying size stratified according to the preoperative lung function in (1) mild COPD or (2) moderate to very severe COPD.

\section{Blinding}

Those blinded to the intervention assignment were: physicians performing the lung function tests, personnel at the intensive care unit (ICU) and ward, personnel performing the 90 days follow-up and the statistician and conclusion drawers. During writing of six abstracts (due to two coprimary outcomes and three comparisons), the intervention groups were identified as 1,2 and 3 and abstracts were approved by all authors before the randomisation code was broken. ${ }^{31}$ Only the operation team was aware of the intervention assignment due to the inherent problem with blinding the surgical perfusion procedure. 


\section{Data collection and verification}

Data for the primary outcome and some of the secondary outcomes were obtained from direct observations and measurements during the hospital stay. Data for the remaining secondary outcomes were obtained at the 90-day follow-up by an in-hospital visit. The primary outcome and serious adverse events (SAE) were verified source data in all patients. Prerandomisation characteristics and secondary outcomes were verified by a random sample of at least $10 \%$ of the patients. All data were registered in case record forms by the trial or clinical personnel under the supervision of the trial investigators. The trial database was established by doubleblinded data entry from the case record forms and subsequently merging of the two databases to minimise typos and errors from converting handwritten data to an electronic database.

\section{Anaesthesia, surgery and protocol interventions}

Anaesthesia was induced using fentanyl $(10 \mu \mathrm{g} / \mathrm{kg})$, propofol $(1-2 \mathrm{mg} / \mathrm{kg})$ and cisatracurium $(0.1 \mathrm{mg} / \mathrm{kg})$ and maintained with sevoflurane $0.5-3 \%$ and continuous infusion of remifentanil $(15-30 \mu \mathrm{g} / \mathrm{kg} /$ hour $)$. Venous access was obtained using two peripheral veins. A sheath for the pulmonary artery catheter and a central line were inserted in one of the internal jugular veins. The ventilation mode was preferentially volume controlled and after CPB positive end expiratory pressure (PEEP) was set to $5 \mathrm{~mm} \mathrm{Hg}$ and lung recruitment performed. Ventilation was ceased during sternotomy and during $\mathrm{CPB}$, irrespective of trial randomisation.

After heparinisation $(350 \mathrm{IU} / \mathrm{kg}$, ACT $>480 \mathrm{~s})$, normothermic CPB (36.5-37.0 ${ }^{\circ} \mathrm{C}$ bladder temperature) was initiated. In the ascending aorta an angled arterial cannula was placed (DLP 24 FR, Medtronic, Minnesota, USA) and, the right atrial appendage was provided with a two-stage venous cannula (36/46 FR, Medtronic, Minnesota, USA). A membrane oxygenator (Capiox RX25, Terumo, Tokyo, Japan) and a roller pump (Stockert S5, Sorin Group, Milano, Italy) were used for perfusion with laminar flow. The arterial line included a $40 \mu \mathrm{m}$ filter (AL06, Pall, New York, USA). Pump flow was $2.4 \mathrm{~L} / \mathrm{min} / \mathrm{m}^{2}$ body surface area and mean arterial pressure was kept between 40 and $70 \mathrm{~mm} \mathrm{Hg}$ by administration of a vasoconstrictor or dilator as appropriate.

For patients in group I and II surgery time was prolonged by no more than $10 \mathrm{~min}$.

In group I (normothermic oxygenated blood): the main pulmonary artery was provided with a straight arterial cannula (14 FR DLP, Medtronic, Minnesota, USA), the aorta was cross-clamped, cardioplegia administered and non-pulsatile continuous pulmonary perfusion was initiated at $300-400 \mathrm{~mL} / \mathrm{min}$. CPB total flow was calculated by DuBois formula (body surface area $\left[\mathrm{m}^{2}\right]=$ Weight $[\mathrm{kg}]^{0.425} \times$ height $\left.(\mathrm{cm})^{0.725} \times 0.007184\right)$ and $10 \%$ was added for the pulmonary perfusion resembling normal physiological conditions. The pulmonary circuit included extra separate roller-pump in the heart- circuit and lung-circuit from where the pulmonary artery perfusion with blood was administered. The tube from the extra roller-pump was connected to a $12 / 14 \mathrm{~F}$ straight arterial cannula placed in the pulmonary artery. The pulmonary artery mean pressure was monitored continuously via a pressure system attached to the side branch of the pulmonary cannula. We aimed to maintain a pulmonary artery flow with a maximum pulmonary artery mean pressure of $20 \mathrm{~mm} \mathrm{Hg}$ resembling the upper limit for normal pulmonary artery pressure. The blood returning from the pulmonary circuit was drained to the venous reservoir via a left atrial vent (VT-53218, CalMed Laboratories, California, USA) inserted through one of the pulmonary veins. Before aortic cross-clamp release, the pulmonary artery perfusion was terminated, the cannula removed and the purse-string suture tied to seal the insertion puncture.

In group II (hypothermic HTK solution): the pulmonary artery was cannulated as described for group I and $2 \mathrm{~L}$ of Custodiol HTK solution at $4^{\circ} \mathrm{C}$ (Dr Franz Köhler Chemie GmbH, Bensheim, Germany) were administered within 8-10 min without pausing the surgical procedure. The pulmonary artery mean pressure was monitored as for group I to guide the infusion rate. The blood and HTK solution returning from the pulmonary circuit (average $1800 \mathrm{~mL}$ ) was directly sucked out from a small hole in the left atrium, filtered by a cell saver, the HTK solution was discarded and the purified red blood cells (average $100 \mathrm{~mL}$ ) returned to the patient via the venous reservoir of the heart-lung machine.

In group III: standard CPB was performed as described above without pulmonary artery perfusion.

The intubation time was defined from end surgery to extubation at the ICU. The patients were extubated when the following criteria were met:

- The patient is awake and alert, pain-free, haemodynamically stable with sufficient cough reflexes and swallowing movements.

- Pressure support ventilation with support $\leq 8 \mathrm{~cm} \mathrm{H} \mathrm{H}_{2} \mathrm{O}$;

- Fraction of inspired oxygen $\left(\mathrm{FiO}_{2} \leq 0,40\right)$;

- Peripheral capillary saturation $\geq 0.94 \%$;

- $\mathrm{pH} \geq 7.34$

- PEEP $\leq 5 \mathrm{~cm} \mathrm{H}_{2} \mathrm{O}$

- Ventilation rate $10-12 / \mathrm{min}$;

- Bleeding from chest drains $\leq 200 \mathrm{~mL} /$ time;

- Bladder temperature $\geq 36^{\circ} \mathrm{C}$.

\section{Outcomes}

The two primary analyses were comparing the two pulmonary perfusion groups with no pulmonary perfusion. Second, a comparison of the two pulmonary perfusion groups was performed. The two coprimary outcomes were the inverse oxygenation index (partial pressure of oxygen $\left(\mathrm{PaO}_{2}\right)$ divided by $\mathrm{FiO}_{2}$ times mean airway pressure) at a single time point 21 hours after start of CPB, and longitudinally at fixed time points $1,3,5,7$ and 21 hours. Reasons for choosing that time points were that (1) haemodynamically stable and extubated patients 
are routinely discharged from the ICU at 21 hours after the start of CPB and (2) the fixed time points $(1,3,5,7$ and 21 hours) approximately correspond to the following surgical events: weaning form $\mathrm{CPB}$, end of surgery, 4 and 6 hours after CPB weaning and 24 hours after anaesthetia induction.

For some of the last oxygenation index measurements the patients were extubated breathing atmospheric air or receiving oxygen supply through a nasal catheter or an oxygen mask. In these cases, the oxygenation index was calculated by first converting the external oxygen supply to an estimated $\mathrm{FiO}_{2},{ }^{32}$ and second by setting the mean airway pressure to 1 , removing that parameter from the equation. For intubated patients respirator settings for $\mathrm{FiO}_{2}$ and mean airway pressure were manually read and noted at the time when blood was drawn from the radial artery and blood gas analysis performed to obtain the $\mathrm{PaO}_{2}$.

The secondary outcomes were: (1) oral tracheal intubation time (hours) after surgery, (2) days alive outside the ICU, (3) days alive outside the hospital, (4) 30-day and 90-day mortality and (5) patients with one or more of the selected SAEs: pneumothorax or pleural effusion requiring drainage, major bleeding, reoperation, severe infection, cerebral event, hyperkalaemia, acute myocardial infarction, cardiac arrhythmia, renal replacement therapy and readmission to the hospital with a respiratory-related condition.

\section{Statistical analysis}

We estimated that a sample size of $3 \times 30$ patients was needed to detect a difference in the $\mathrm{PF}\left(\mathrm{PaO}_{2} / \mathrm{FiO}_{2}\right)$ ratio of $55 \mathrm{~mm} \mathrm{Hg}$ for the group perfused with oxygenated blood compared with no pulmonary perfusion with a power of $90 \%$ and a two-sided maximal risk of type 1 error of $0.025 .^{27} 29$ The PF ratio was used for the power calculation as no clinical trials in adults had the oxygenation index as an outcome measure. The oxygenation index includes lung compliance in the equation, a clinically important parameter for intubated patient's oxygenation, and therefore we chose it as our primary outcome instead of the PF ratio.

The primary analysis included a modified intention-to-treat population defined as all randomised patients, except patients not receiving CPB-dependent cardiac surgery, and a per-protocol population excluding patients with major protocol violations defined as: (1) patients who were randomised to an intervention but did not receive any intervention and (2) patients who received an incorrect intervention. For the coprimary outcomes all analyses were adjusted for the stratification variable (mild COPD or moderate to very severe COPD) and the patients' baseline oxygenation index. We then adjusted for both the stratification and design variables (age, $\mathrm{FEV}_{1}$, left ventricular ejection fraction and the patients' baseline oxygenation index).$^{29}$

Linear regression was used to compare the three groups' oxygenation indices at the last time point
21 hours after CPB start. Linear mixed-effects model (MEM) analysis was used to analyse differences in the longitudinal measurements of the oxygenation indices between the three groups.

Proportions of patients with one or more SAEs and 30 days and 90 days mortality were analysed as dichotomous variables using logistic regression adjusted for the stratification variable. The remaining secondary outcomes were count data analysed with the van Elteren test $^{33}$ and were therefore only adjusted for the stratification variable.

We will assess the validity of the trial results according to a five-point procedure which includes adjustments of thresholds for significance according to the number of primary outcome comparisons. We will use a $\mathrm{p}$ value threshold for significance of $0.025(0.05 / 2$ because two primary outcome comparisons are used) and a Bayes factor threshold for significance of 0.1. ${ }^{34}$

\section{RESULTS}

\section{Patients}

A total of 3688 patients were screened and 387 patients meet the inclusion criteria. Ninety patients were randomised due to trial limitations of only two patients per week. Thirty patients were randomised to pulmonary artery perfusion with normothermic oxygenated blood, 29 to pulmonary artery perfusion with hypothermic HTK solution and 31 to no pulmonary artery perfusion. Eighty-nine of the patients received the intervention and were included in the intention-to-treat analysis of the primary outcome and 86 patients were included in the per-protocol analysis. The 90-day follow-up for survival was complete for 89 patients (figure 1 and see online supplementary table S1).

The baseline characteristics of the patients, comorbidities, preoperative pulmonary status and surgical data are summarised in table 1 . Mean age was 70 years, and $75 \%$ were men. We found no statistically significant differences for the baseline characteristics between the three groups.

\section{Outcomes}

Our primary comparisons were (1) pulmonary artery perfusion with normothermic oxygenated blood compared with no pulmonary perfusion and (2) pulmonary artery perfusion with hypothermic HTK solution compared with no pulmonary perfusion. For the oxygenation index we present results for both the last measurement at 21 hours and the longitudinal measurements. The inverse unadjusted mean oxygenation indices are illustrated in figure 2 and listed in table 2 which also includes the unadjusted mean PF ratios (not inversed oxygenation indices see online supplementary table S2).

For the PF ratio the two perfused groups demonstrates their lowest PF ratio 1 hour after CPB start with the HTK group showing the most pronounced deterioration of all three groups. For the group receiving no pulmonary 


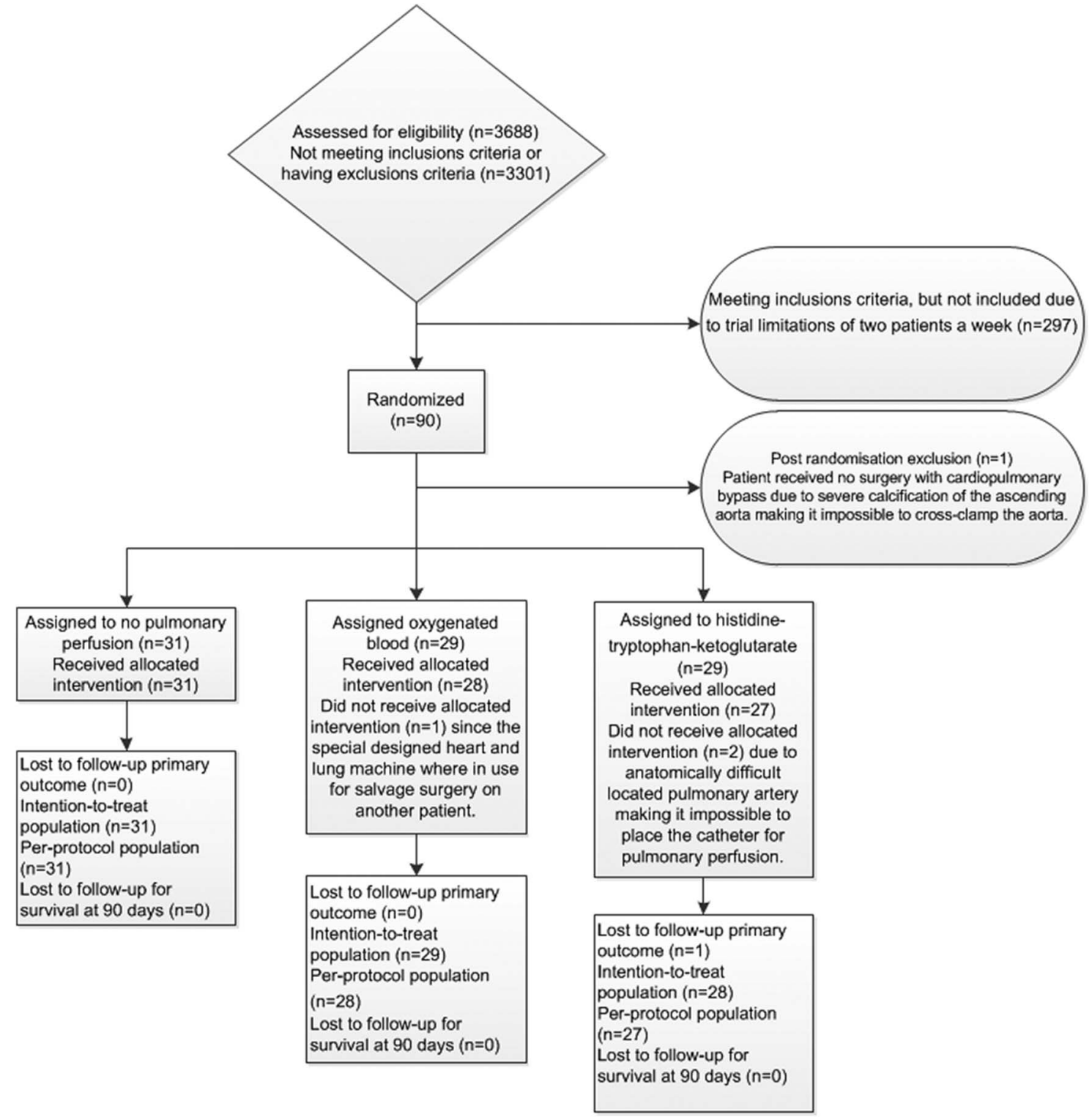

Figure 1 CONSORT flow diagram.

perfusion the lowest PF ratio was found 3 hours after CPB start. Point estimates for this group demonstrate the greatest overall change in $\mathrm{PF}$ ratio from baseline to 21 hours of $122 \mathrm{~mm} \mathrm{Hg}$, compared to only 61 and $84 \mathrm{~mm} \mathrm{Hg}$ for the groups receiving oxygenated blood and HTK solution, respectively.

At 21 hours after CPB start, patients who received pulmonary artery perfusion with oxygenated blood had a higher oxygenation index compared to patients receiving no pulmonary artery perfusion during $\mathrm{CPB}$ (mean difference (MD) $0.94 ; 95 \%$ CI 0.05 to $1.83 ; \mathrm{p}=0.04$ ). This corresponds to a difference in the $\mathrm{PaO}_{2}$ of $22 \mathrm{~mm} \mathrm{Hg}$ with a median $\mathrm{FiO}_{2}$ of 0.32 . The oxygenation index was also significantly higher at 21 hours (MD 0.99; CI 0.29 to $1.69 ; \mathrm{p}=0.007)$ and longitudinally ((MEM, $\mathrm{p}=0.009$ ) for patients who received pulmonary artery perfusion with oxygenated blood compared to pulmonary artery perfusion with HTK solution. This corresponds to a difference in the $\mathrm{PaO}_{2}$ of $23 \mathrm{~mm} \mathrm{Hg}$ with a median $\mathrm{FiO}_{2}$ of 0.32 (see online supplementary table S3).

We found no statistically significant differences in the longitudinal measurements of the oxygenation index between patients who received pulmonary artery perfusion with oxygenated blood or HTK solution compared with no pulmonary perfusion (MEM, $\mathrm{p}=0.57$ and $\mathrm{p}=0.17$, respectively). The same applied at 21 hours for the patients who received pulmonary artery perfusion with HTK solution compared with no pulmonary perfusion (MD $0.06 ; 95 \%$ CI -0.73 to $0.86 ; \mathrm{p}=0.87$ ). The secondary and per-protocol analyses did not noticeably change the results of the coprimary outcome (see online supplementary table S4).

We found no statistically significant differences between the groups for any of the secondary outcomes (table 3 and see online supplementary table S5) although point estimates (except for SAEs), favoured 
Table 1 Characteristics of the patients at baseline and surgical data*

\begin{tabular}{|c|c|c|c|}
\hline Characteristics & $\begin{array}{l}\text { Oxygenated } \\
\text { blood } \\
(\mathrm{N}=29)\end{array}$ & $\begin{array}{l}\text { HTK solution } \\
(\mathrm{N}=29)\end{array}$ & $\begin{array}{l}\text { No pulmonary } \\
\text { perfusion } \\
(\mathrm{N}=31)\end{array}$ \\
\hline \multicolumn{4}{|l|}{ Demographic characteristics } \\
\hline Age-year & $69.9 \pm 10.8$ & $69.6 \pm 8.0$ & $70.5 \pm 8.9$ \\
\hline Male sex-number (\%) & $22(75.9)$ & $20(69.0)$ & $25(80.6)$ \\
\hline \multicolumn{4}{|c|}{ Comorbidities-number/total number (\%) (number of patients with missing values) } \\
\hline Self-reported COPD & $15 / 29(51.7)$ & $18 / 29(62.1)$ & 19/31 (61.3) \\
\hline $\begin{array}{l}\text { Pulmonary hypertension determined by preoperative } \\
\text { echocardiographic }\end{array}$ & $3 / 29(10.3)(5)$ & $7 / 29(24.1)(3)$ & $6 / 31(19.4)(8)$ \\
\hline Arterial hypertension & 23/29 (79.3) & $24 / 29(82.8)$ & $22 / 31(71.0)$ \\
\hline Chronic atrial fibrillation or flutter & $4 / 29(13.8)$ & $3 / 29(10,3)[1]$ & $5 / 31(16.1)$ \\
\hline Chronic heart failure $\dagger$ & $13 / 29(44.8)$ & $6 / 29(20.7)[2]$ & 9/31 (29.0) \\
\hline ASA class IV or worse & $1 / 29(3.4)$ & $4 / 29(13.8)[2]$ & $4 / 31(12.9)[2]$ \\
\hline Median left ventricular ejection fraction (IQR) & $52.5(41.2-60.0)[1]$ & $50.0(37.5-60.0)$ & $55.0(45.0-60.0)$ \\
\hline Recent AMI‡ & $7 / 29(24.1)$ & $11 / 29(37.9)$ & $7 / 31(22.6)$ \\
\hline Insulin-dependent diabetes mellitus & $3 / 29(10.3)$ & $3 / 29(10.3)$ & $2 / 31(6.5)$ \\
\hline Median estimated creatinine clearance (IQR) $§$ & $82.5(64.2-121.9)$ & $82.7(62.8-107.3)$ & $86.1(65.2-106.8)$ \\
\hline Previous transit ischaemic attack or stroke & $1 / 29(3.4)$ & $7 / 29(24.1)$ & $7 / 31(22.6)$ \\
\hline Previous percutaneous coronary intervention & $11 / 29(37.9)$ & $7 / 29(24.1)$ & $7 / 31(22.6)$ \\
\hline Implantable cardioverter defibrillator or pacemaker & $2 / 29(6.9)[2]$ & $1 / 29(3.4)[1]$ & $1 / 31(3.2)[1]$ \\
\hline Greater alcohol consumptionף & $11 / 29(37.9)$ & $7 / 29(24.1)$ & $7 / 31(22.6)$ \\
\hline Median tobacco pack years (IQR) & $40.0(18.5-51.0)$ & $45.0(32.5-50.0)$ & $35.0(20.0-45.0)$ \\
\hline \multicolumn{4}{|l|}{ Pulmonary function } \\
\hline $\begin{array}{l}\text { Median per cent predicted forced } \\
\text { expiratory volume in } 1 \mathrm{~s} .(\mathrm{IQR})\end{array}$ & $75.0(56.5-87.5)$ & $79.0(58.5-88.0)$ & $74.0(58.0-88.0)$ \\
\hline GOLD classification Mild & $14 / 29(48.3)$ & $14 / 29(48.3)$ & $12 / 31(38.7)$ \\
\hline GOLD classification Moderate & $11 / 29(37.9)$ & $14 / 29(48.3)$ & $17 / 29(54.8)$ \\
\hline GOLD classification Severe & $3 / 29(10.3)$ & $1 / 29(3.4)$ & $2 / 31(6.5)$ \\
\hline GOLD classification Very severe & $1 / 29(3.5)$ & $0 / 29(0.0)$ & $0 / 29(0.0)$ \\
\hline \multicolumn{4}{|l|}{ Surgical data } \\
\hline Elective surgery & $18 / 29(62.1)$ & $16 / 29(55.2)$ & 23/31 (74.2) \\
\hline Urgent surgery & $11 / 29(37.9)$ & $13 / 29(44.8)$ & $8 / 31(25.8)$ \\
\hline \multicolumn{4}{|l|}{ Type of surgery } \\
\hline AVR & $7 / 29(24.1)$ & $7 / 29(24.1)$ & $12 / 31(38.7)$ \\
\hline CABG & $15 / 29(51.7)$ & $17 / 29(58.6)$ & $17 / 31(54.8)$ \\
\hline CABG and AVR & $7 / 29(24.1)$ & $5 / 29(17.2)$ & $2 / 31(6.5)$ \\
\hline Cardiopulmonary bypass time (min) & $102.2 \pm 53.7$ & $100.9 \pm 26.5$ & $98.45 \pm 44.6$ \\
\hline Aortic cross-clamp time (min) & $58.8 \pm 37.9$ & $64.3 \pm 18.3$ & $61.9 \pm 34.7$ \\
\hline
\end{tabular}

*Plus-minus values are means \pm SD. There were no statistical significant differences between the three groups for any of the variables.

tChronic heart failure was defined as New York Heart Association Class III or worse.

$\ddagger$ Recent $\mathrm{AMI}$ is within 3 months prior to the surgery.

§stimated creatinine clearance by Cockcroft-Gault equation in $\mathrm{mL} / \mathrm{min} / 1.73 \mathrm{~m}^{2}$.

ๆGreater alcohol consumption was defined as $>14$ for women and $>21$ for men units' alcohol per week.

AMI, acute myocardial infarction; ASA, American Society of Anesthesiologists; AVR, aortic valve replacement; CABG, coronary artery bypass graft;

COPD, chronic obstructive pulmonary disease; GOLD, global initiative for chronic obstructive lung disease; HTK, histidine-tryptophan-ketoglutarate.

pulmonary artery perfusion with oxygenated blood compared with no pulmonary perfusion.

The proportions of patients with one or more of the selected SAEs did not differ between the three groups. The number of patients with 0-6 SAEs are listed in online supplementary table $\mathrm{S} 6$ and described in details in online supplementary table S7, both in the online supplementary appendix. There were no serious adverse reactions or suspected unexpected serious adverse reactions for any of the trial patients.

Within the total observation time from randomisation of the first patient and until 90 days after randomisation of the last patient (90-574 days) $8(9 \%)$ patients had died with an equal distribution in the three groups ( 3 in the blood group, 3 in HTK group and 2 in the no pulmonary perfusion group). The relative high mortality probably reflects that the trial population is elderly and with a high prevalence of comorbidities.

\section{DISCUSSION}

In this randomised clinical trial we tested in a clinical setting (1) pulmonary artery perfusion with normothermic oxygenated blood or (2) cooling of the lungs by pulmonary artery perfusion with hypothermic HTK solution compared with (3) no pulmonary artery perfusion 
during CPB in cardiac surgery patients with COPD. At 21 hours after starting $\mathrm{CPB}$, patients perfused with oxygenated blood had a significantly higher oxygenation index compared with those receiving no pulmonary perfusion. However, the $p$ value was above our predefined significance level $(0.025)$ based on multiple comparisons. ${ }^{34}$ The oxygenated blood group had also a significantly higher oxygenation index on both the longitudinal measurements and at 21 hours compared with the HTK group. The trial was not powered to

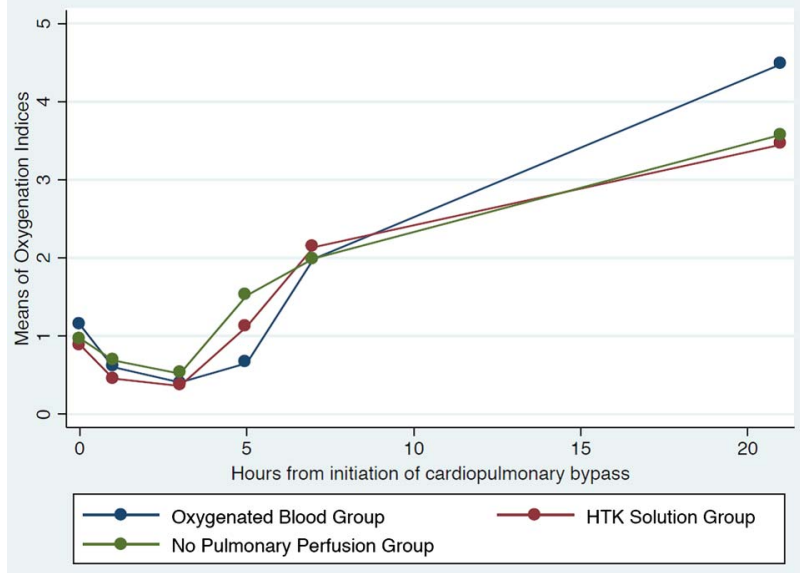

Figure 2 Oxygenation indices inverse means for the three groups from cardiopulmonary bypass start and until 21 hours after. detect clinically relevant differences and therefore we found no significant differences between the three groups in regards to tracheal intubation time, SAEs, mortality or days alive outside the ICU and outside the hospital.

As reported in animal studies, ${ }^{13-16}$ small randomised trials and observational studies in infants ${ }^{18} 19$ and adults ${ }^{22-25}$ we have now evaluated and confirmed in a clinical setting with adult patients with COPD, that pulmonary artery perfusion with oxygenated blood during CPB results in a higher postoperative oxygenation. This was obtained without an increased incidence of complications or significant prolongation of the surgical procedure. However, we could not confirm the findings of a higher postoperative oxygenation in patients receiving pulmonary artery perfusion with hypothermic Custodiol HTK solution. ${ }^{15} 172021$

The severity of postoperative pulmonary dysfunction is related to the patients' preoperative pulmonary function and reduced lung function is associated with increased morbidity and mortality ${ }^{35-37}$ Patients with reduced lung function like COPD therefore face a multitude of problems during and after surgery, as cardiac surgery is offered to an increasingly elderly and sicker population, with a significant age-related decline in respiratory reserves. ${ }^{38-40}$ All patients developed reduced PF-ratio and had, according to the recent Berlin definition, ${ }^{41}$ a mild degree of ARDS with PF-ratios between 200 and $300 \mathrm{~mm} \mathrm{Hg}$ in the perioperative 24 hours (table 2). In

Table 2 Unadjusted inverse oxygenation indices and PF ratio*

\begin{tabular}{|c|c|c|}
\hline & Ol mean $(95 \% \mathrm{Cl})$ & PF mean $(95 \% \mathrm{Cl})$ \\
\hline \multicolumn{3}{|l|}{ Baseline } \\
\hline Pulmonary perfusion with oxygenated blood & 1.15 (0.78 to 1.52$) 0.89$ & 392.35 (348.06 to 436.64$)$ \\
\hline Pulmonary perfusion with HTK solution & $(0.74$ to 1.04$)$ & 350.14 (314.80 to 385.48$)$ \\
\hline No pulmonary perfusion & $0.97(0.83$ to 1.11$)$ & 413.43 (371.37 to 455.49$)$ \\
\hline \multicolumn{3}{|l|}{1 hour after CPB start } \\
\hline Pulmonary perfusion with oxygenated blood & $0.60(0.48$ to 0.73$)$ & 272.00 (225.90 to 318.09$)$ \\
\hline Pulmonary perfusion with HTK solution & $0.46(0.32$ to 0.59$)$ & 199.96 (160.15 to 239.77$)$ \\
\hline No pulmonary perfusion & $0.69(0.55$ to 0.83$)$ & 293.02 (245.93 to 340.12$)$ \\
\hline \multicolumn{3}{|l|}{3 hours after CPB start } \\
\hline Pulmonary perfusion with oxygenated blood & $0.41(0.33$ to 0.48$)$ & 282.11 (237.46 to 326.76$)$ \\
\hline Pulmonary perfusion with HTK solution & $0.36(0.30$ to 0.42$)$ & 249.64 (214.58 to 284.70$)$ \\
\hline No pulmonary perfusion & $0.52(0.27$ to 0.77$)$ & 267.84 (216.66 to 319.03$)$ \\
\hline \multicolumn{3}{|l|}{5 hours after CPB start } \\
\hline Pulmonary perfusion with oxygenated blood & $0.65(0.37$ to 0.94$)$ & 337.15 (260.56 to 413.74$)$ \\
\hline Pulmonary perfusion with HTK solution & $1.12(0.55$ to 1.69$)$ & 262.86 (235.55 to 290.18$)$ \\
\hline No pulmonary perfusion & $1.52(0.67$ to 2.37$)$ & $285.84(238.32$ to 333.36$)$ \\
\hline \multicolumn{3}{|l|}{7 hours after CPB start } \\
\hline Pulmonary perfusion with oxygenated blood & 1.99 (1.20 to 2.77$)$ & $321.00(286.47$ to 355.54$)$ \\
\hline Pulmonary perfusion with HTK solution & 2.14 (1.46 to 2.82$)$ & 274.82 (250.64 to 299.11$)$ \\
\hline No pulmonary perfusion & 1.99 (1.23 to 2.76$)$ & 277.43 (239.55 to 315.32$)$ \\
\hline \multicolumn{3}{|l|}{21 hours after CPB start } \\
\hline Pulmonary perfusion with oxygenated blood & 4.48 (3.86 to 5.10$)$ & 331.04 (285.54 to 376.54$)$ \\
\hline Pulmonary perfusion with HTK solution & 3.45 (3.06 to 3.84$)$ & $266.03(238.91$ to 293.15$)$ \\
\hline No pulmonary perfusion & 3.58 (2.93 to 4.22$)$ & 291.37 (254.58 to 328.16$)$ \\
\hline
\end{tabular}

*Intention-to-treat population.

$\mathrm{CPB}$, cardiopulmonary bypass; $\mathrm{HTK}$, histidine-tryptophan-ketoglutarate; OI, oxygenation index; $\mathrm{PF}, \mathrm{PaO}_{2} / \mathrm{FiO}_{2}$ ratio in $\mathrm{mm} \mathrm{Hg}$. 
Table 3 Effects of pulmonary artery perfusion versus no pulmonary perfusion on the secondary outcomes*

p Value

Oral tracheal intubation time †

Oxygenated blood vs no pulmonary perfusion 0.47

HTK vs no pulmonary perfusion $\quad 0.10$

Oxygenated blood vs HTK solution 0.23

Days alive outside ICU †

Oxygenated blood vs no pulmonary perfusion 0.42

HTK vs no pulmonary perfusion 0.61

Oxygenated blood vs HTK solution 0.92

Days alive outside the hospital $\dagger$

Oxygenated blood vs no pulmonary perfusion 0.86

HTK vs no pulmonary perfusion $\quad 0.99$

Oxygenated blood vs HTK solution $\quad 0.97$

\begin{tabular}{lll}
\hline & OR (95\% CI) & p Value \\
\hline Death at 90 days $\ddagger$ & & \\
Oxygenated blood vs no pulmonary perfusion & $0.69(0.09$ to 3.10$)$ & 0.98 \\
HTK vs no pulmonary perfusion & $0.78(0.01$ to 65.37$)$ & 1.00 \\
Oxygenated blood vs HTK solution & $0.47(0.01$ to 9.33$)$ & \\
Patients with one or more serious adverse events $\ddagger$ & & 0.96 \\
Oxygenated blood vs no pulmonary perfusion & $0.79(0.19$ to 3.30$)$ \\
HTK vs no pulmonary perfusion & $0.61(0.13$ to 2.85$)$ \\
Oxygenated blood vs HTK solution & $1.33(0.27$ to 6.53$)$ & 0.75 \\
\hline
\end{tabular}

${ }^{*}$ Analysis of the intention-to-treat population.

†Van Elteren test.

†Exact logistic regression adjusted for stratification variable.

HTK, histidine-tryptophan-ketoglutarate; ICU, intensive care unit; ITT, intension to treat.

this respect, it is interesting that the highest PF-ratio at baseline was seen in the control group, which was also the group demonstrating the most pronounced deterioration over the next 21 hours with a delta-difference in PF-ratio from baseline to 21 hours of $122 \mathrm{~mm} \mathrm{Hg}$, compared to only 61 and $84 \mathrm{~mm} \mathrm{Hg}$ for the groups receiving oxygenated blood and HTK solution, respectively.

Our trial had a number of strengths. The protocol was pragmatic so routine practice was maintained, and we predefined the methodology in a protocol, and a detailed statistical analysis plan, which minimised the risk of datadriven analyses and outcome reporting bias. ${ }^{42}$ Additional strengths were the authors' unawareness of the randomisation groups during the statistical analyses and writing of six abstracts, which were approved by all authors before the blinding was unveiled.

The limitations of our trial were mainly that the primary outcome (oxygenation index) was a surrogate outcome with unknown long-term clinical relevance for the patients. We chose the oxygenation index, and not $\mathrm{PF}$ ratio, as it includes the mean airway pressure in the calculation which can be an important correcting parameter for oxygenation monitoring in mechanically ventilated patients with excessive pulmonary dysfunction. Second, we chose our primary comparison to be pulmonary perfusion with oxygenated blood or HTK solution compared with no pulmonary perfusion. Comparing the two pulmonary perfusion techniques were considered explorative, but came out with the strongest significant result. Third, we had limited power to detect any differences in the secondary outcomes, probably due to a small sample size. We believe that a larger multicentre randomised trial, including patients with both reduced and normal lung function undergoing cardiac surgery, could provide conclusive results on the short-term and long-term clinical benefits of pulmonary artery perfusion with oxygenated blood during CPB. Based on our results we cannot encourage further exploration of pulmonary artery perfusion with hypothermic Custodiol HTK solution during CPB.

In conclusion we found that pulmonary artery perfusion with normothermic oxygenated blood during CPB resulted in improved postoperative oxygenation for patients with COPD undergoing cardiac surgery compared with both no pulmonary perfusion and pulmonary artery perfusion with hypothermic HTK solution. For those patients receiving hypothermic HTK solution postoperative oxygenation did not improve.

\section{Author affiliations}

${ }^{1}$ Department of Cardiothoracic Anesthesiology, The Heart Centre, Rigshospitalet, Copenhagen, Denmark

${ }^{2}$ Department of Biomedical Sciences, Panum inst, University of Copenhagen, Copenhagen, Denmark

${ }^{3}$ Department of Anesthesiology, Rigshospitalet, Copenhagen, Denmark

${ }^{4}$ Department of Cardiothoracic Surgery, The Heart Centre, Rigshospitalet, Copenhagen, Denmark

${ }^{5}$ The Copenhagen Trial Unit, Centre for Clinical Intervention Research,

Rigshospitalet, Copenhagen, Denmark

${ }^{6}$ Department of Cardiology, Holbæk Hospital, Denmark 
Acknowledgements The authors wish to thank the staff at the Department of Cardiothoracic Anaesthesiology and Surgery at Rigshospitalet, Copenhagen University Hospital, Denmark for supporting the trial. The authors also wish to thank the following: Dr Liu Ya Ling and Christian Secher for their help with acquisition and handling of data; the perfusionists for their invaluable theoretical and practical help while running perfusions on the trial patients; the Department of Clinical Biochemistry, Rigshospitalet, Copenhagen University Hospital, Denmark; the Lundbeck Foundation, Ehrenreich Foundation, Aase \& Ejnar Danielsen Foundation and Moller Foundation, Pharmanovia A/S and the Heart Centre Research Committee at Rigshospitalet, Copenhagen University Hospital, Denmark.

Contributors KBB drafted the manuscript. KBB, JCW, TJ, LWA and DAS contributed to the conception and design of the trial. KBB and MMS were responsible for the acquisition of data. KBB, JCJ and JW executed the statistical analyses and interpretation of the data. KBB, NHS, HBR, JCJ and JW contributed with drafting of the article and critical revision for important intellectual content. All authors revised, amended and approved the final manuscript.

Funding The work was supported by the Lundbeck Foundation R100-A9676; Pharmanovia A/S; the Aase \& Ejnar Danielsen Foundation; the AP Moeller Foundation; the Ehrenreich Foundation and by the Heart Centre Research Committee at Rigshospitalet, University of Copenhagen, Denmark.

Competing interests KBB declares to have received an unrestricted grant from the Lundbeck Foundation not involved in producing histidine-tryptophanketoglutarate solution. In addition, KBB has received an unrestricted grant by Nordmedica A/S (now Pharmanovia A/S) distributing HTK solution. The grant was awarded in January 2012 and used for 4 months' salary (200 000 DKR of the total budget, 3.2 million DKR) to write the protocol for the Pulmonary Protection Trial.

Ethics approval The Pulmonary Protection Trial was approved by the Committees on Biomedical Research Ethics of The Capital Region of Denmark, the Danish Medicines Agency, the Danish Data Protection Agency and registered at ClinicalTrials.gov (6 June 2012, NCT01614951, Principal Investigator DAS).

Provenance and peer review Not commissioned; externally peer reviewed.

Data sharing statement No additional data are available.

Open Access This is an Open Access article distributed in accordance with the Creative Commons Attribution Non Commercial (CC BY-NC 4.0) license, which permits others to distribute, remix, adapt, build upon this work noncommercially, and license their derivative works on different terms, provided the original work is properly cited and the use is non-commercial. See: http:// creativecommons.org/licenses/by-nc/4.0/

\section{REFERENCES}

1. LaPar DJ, Gillen JR, Crosby IK, et al. Predictors of operative mortality in cardiac surgical patients with prolonged intensive care unit duration. J Am Coll Surg 2013;216:1116-23.

2. Kaul TK, Fields BL, Riggins LS, et al. Adult respiratory distress syndrome following cardiopulmonary bypass: incidence, prophylaxis and management. J Cardiovasc Surg (Torino) 1998;39:777-81.

3. Koch $\mathrm{C}$, Li L, Figueroa $\mathrm{P}$, et al. Transfusion and pulmonary morbidity after cardiac surgery. Ann Thorac Surg 2009;88:1410-18.

4. Milot J, Perron J, Lacasse $\mathrm{Y}$, et al. Incidence and predictors of ARDS after cardiac surgery. Chest 2001;119:884-8.

5. Christenson JT, Aeberhard J-M, Badel P, et al. Adult respiratory distress syndrome after cardiac surgery. Cardiovasc Surg 1996;4:15-21.

6. Massoudy P, Zahler S, Becker BF, et al. Evidence for inflammatory responses of the lungs during coronary artery bypass grafting with cardiopulmonary bypass. Chest 2001;119:31-6.

7. Schlensak C, Doenst T, Beyersdorf F. Lung ischemia during cardiopulmonary bypass. Ann Thorac Surg 2000;70:337-8.

8. den Hengst WA, Gielis JF, Lin JY, et al. Lung ischemia-reperfusion injury: a molecular and clinical view on a complex pathophysiological process. Am J Physiol Heart Circ Physiol 2010;299:H1283-99.

9. Chai PJ, Williamson JA, Lodge AJ, et al. Effects of ischemia on pulmonary dysfunction after cardiopulmonary bypass. Ann Thorac Surg 1999;67:731-5.
10. Friedman M, Sellke FW, Wang SY, et al. Parameters of pulmonary injury after total or partial cardiopulmonary bypass. Circulation 1994;90Pt 2):II262-268.

11. Schlensak C, Doenst T, Preusser S, et al. Bronchial artery perfusion during cardiopulmonary bypass does not prevent ischemia of the lung in piglets: assessment of bronchial artery blood flow with fluorescent microspheres. Eur J Cardiothorac Surg 2001;19: 326-32.

12. Kuratani $\mathrm{T}$, Matsuda $\mathrm{H}$, Sawa $\mathrm{Y}$, et al. Experimental study in a rabbit model of ischemia-reperfusion lung injury during cardiopulmonary bypass. J Thorac Cardiovasc Surg 1992;103:564-8.

13. da Costa Freitas CR, Sa Malbouisson LM, Benicio A, et al. Lung perfusion and ventilation during cardiopulmonary bypass reduces early structural damage to pulmonary parenchyma. Anesth Analg 2016;122:943-52.

14. Grossherr M, Bechtel JFM, Heinze $\mathrm{H}$, et al. Effects of pulmonary artery perfusion on gas exchange and alveolar matrix metalloproteinases after cardiopulmonary bypass in a swine model. App Cardiopulm Pathophysiol 2009;13:123-9.

15. Serraf $A$, Robotin M, Bonnet $N$, et al. Alteration of the neonatal pulmonary physiology after total cardiopulmonary bypass. $J$ Thorac Cardiovasc Surg 1997;114:1061-9.

16. Zheng $\mathrm{JH}, \mathrm{Xu} \mathrm{ZW}$, Wang $\mathrm{W}$, et al. Lung perfusion with oxygenated blood during aortic clamping prevents lung injury. Asian Cardiovasc Thorac Ann 2004;12:58-60.

17. Li JA, Liu YL, Liu JP, et al. Pulmonary artery perfusion with HTK solution prevents lung injury in infants after cardiopulmonary bypass. Chin Med J 2010;123:2645-50.

18. Suzuki T, Fukuda $\mathrm{T}$, Ito $\mathrm{T}$, et al. Continuous pulmonary perfusion during cardiopulmonary bypass prevents lung injury in infants. Ann Thorac Surg 2000;69:602-6.

19. Suzuki T, Ito T, Kashima I, et al. Continuous perfusion of pulmonary arteries during total cardiopulmonary bypass favorably affects levels of circulating adhesion molecules and lung function. $J$ Thorac Cardiovasc Surg 2001;122:242-8.

20. Wei B, Liu $Y$, Wang $Q$, et al. Lung perfusion with protective solution relieves lung injury in corrections of Tetralogy of Fallot. Ann Thorac Surg 2004;77:918-24

21. De Santo LS, Romano G, Amarelli C, et al. Surgical repair of acute type A aortic dissection: continuous pulmonary perfusion during retrograde cerebral perfusion prevents lung injury in a pilot study. J Thorac Cardiovasc Surg 2003;126:826-31.

22. Kiessling $A H$, Guo FW, Gökdemir $Y$, et al. The influence of selective pulmonary perfusion on the inflammatory response and clinical outcome of patients with chronic obstructive pulmonary disease undergoing cardiopulmonary bypass. Interact Cardiovasc Thorac Surg 2014;18:732-9.

23. Santini F, Onorati F, Telesca M, et al. Selective pulmonary pulsatile perfusion with oxygenated blood during cardiopulmonary bypass attenuates lung tissue inflammation but does not affect circulating cytokine levels. Eur J Cardiothorac Surg 2012;42:942-50.

24. Santini F, Onorati F, Telesca M, et al. Pulsatile pulmonary perfusion with oxygenated blood ameliorates pulmonary hemodynamic and respiratory indices in low-risk coronary artery bypass patients. Eur J Cardiothorac Surg 2011;40:794-803.

25. Liu LM, Hu JG, Yin BL, et al. [Lung protection of continuous pulmonary artery perfusion with oxygenated blood during cardiopulmonary bypass]. [Chinese]. Zhong Nan Da Xue Xue Bao Yi Xue Ban 2005;30:413-16.

26. Griffith BP, Zenati M. The pulmonary donor. Clin Chest Med 1990;11:217-26

27. Buggeskov KB, Wetterslev J, Secher NH, et al. Pulmonary perfusion with oxygenated blood or custodiol HTK solution during cardiac surgery for postoperative pulmonary function in COPD patients: a trial protocol for the randomized, clinical, parallel group, assessor and data analyst blinded Pulmonary Protection Trial. Trials 2013;14:30.

28. World Medical Association. World Medical Association Declaration of Helsinki: ethical principles for medical research involving human subjects. JAMA 2013;310:2191-4.

29. Buggeskov KB, Jakobsen JC, Secher NH, et al. Detailed statistical analysis plan for the pulmonary protection trial. Trials 2014;15:510.

30. GOLD Report 2016.pdf. [cited 2 Feb 2016]. http://www.goldcopd. org/uploads/users/files/GOLD_Report\%202016.pdf

31. Gøtzsche PC. Blinding during data analysis and writing of manuscripts. Control Clin Trials 1996;17:285-90-293.

32. Waldau T, Larsen VH, Bonde J. Evaluation of five oxygen delivery devices in spontaneously breathing subjects by oxygraphy. Anaesthesia 1998:53:256-63.

33. Jakobsen JC, Tamborrino M, Winkel P. Count data analysis in randomised clinical trials. J Biomet Biostat 2015[cited 2015 Nov 18];6:227. http://www.omicsonline.org/open-access/count-data- 
analysis-in-randomised-clinical-trials-2155-6180-1000227.php? aid $=54594$

34. Jakobsen JC, Gluud C, Winkel P, et al. The thresholds for statistical and clinical significance-a five-step procedure for evaluation of intervention effects in randomised clinical trials. BMC Med Res Methodol 2014;14:34.

35. Adabag AS, Wassif HS, Rice K, et al. Preoperative pulmonary function and mortality after cardiac surgery. Am Heart J 2010;159:691-7.

36. Ried M, Unger P, Puehler T, et al. Mild-to-moderate COPD as a risk factor for increased 30-day mortality in cardiac surgery. Thorac Cardiovasc Surg 2010;58:387-91.

37. Roques F, Nashef SA, Michel P, et al. Risk factors and outcome in European cardiac surgery: analysis of the EuroSCORE multinational database of 19030 patients. Eur J Cardiothorac Surg 1999;15:816-23.
38. Fuster RG, Argudo JAM, Albarova OG, et al. Prognostic value of chronic obstructive pulmonary disease in coronary artery bypass grafting. Eur J Cardiothorac Surg 2006;29:202-9.

39. Leavitt BJ, Ross CS, Spence B, et al. Long-term survival of patients with chronic obstructive pulmonary disease undergoing coronary artery bypass surgery. Circulation 2006;114(1 Suppl):1430-434.

40. Manganas $\mathrm{H}$, Lacasse $\mathrm{Y}$, Bourgeois $\mathrm{S}$, et al. Postoperative outcome after coronary artery bypass grafting in chronic obstructive pulmonary disease. Can Respir J 2007;14:19-24.

41. ARDS Definition Task ForceRanieri VM, Rubenfeld GD, Thompson $\mathrm{BT}$, et al. Acute respiratory distress syndrome: the Berlin Definition. JAMA 2012;307:2526-33.

42. Thomas $L$, Peterson ED. The value of statistical analysis plans in observational research: defining high-quality research from the start. JAMA 2012;308:773-4. 